\title{
Effect of Flexible 3D Multipoint Stretch Bending Dies on the Shape Accuracy and the Optimal Design
}

\author{
Xiangfeng Lin $\mathbb{D}^{1},{ }^{1,2}$ Yi Li $\mathbb{D}^{1,3}$ Zhongyi Cai, ${ }^{1,2}$ Jicai Liang, ${ }^{1,2}$ Ce Liang, ${ }^{1,3}$ Kaifeng Yu $\mathbb{D}^{1,3}$ \\ Xiaoming $L i \mathbb{D}^{1,3}$ Aicheng Wang $\left(\mathbb{D},{ }^{1,2}\right.$ and Yanfei Liao $\mathbb{D}^{1,2}$ \\ ${ }^{1}$ Key Laboratory of Automobile Materials, Ministry of Education, Jilin University, Changchun, Jilin 130025, China \\ ${ }^{2}$ Roll Forging Institute, Jilin University, Changchun, Jilin 130025, China \\ ${ }^{3}$ College of Materials Science and Engineering, Jilin University, Changchun, Jilin 130025, China
}

Correspondence should be addressed to Yi Li; henrylee@jlu.edu.cn

Received 16 January 2018; Revised 20 March 2018; Accepted 25 March 2018; Published 7 May 2018

Academic Editor: Hongchao Kou

Copyright $(92018$ Xiangfeng Lin et al. This is an open access article distributed under the Creative Commons Attribution License, which permits unrestricted use, distribution, and reproduction in any medium, provided the original work is properly cited.

Aiming at the flexible 3D stretch bending with multipoint dies for the aluminum profile, the numerical simulation analysis of the bending process was carried out by ABAQUS finite element software. In the multipoint stretch bending (MPSB) process, the influence of the number of die units on the springback for the complex section profile was studied. The shape error between the forming parts and the target parts was reduced through the method of die surface modification. The results showed that the springback of the aluminum profile could reach a minimum when the number of die units was 25 under the precondition of saving cost and ensuring the quality of forming parts. In the numerical simulation, the maximum shape error of the forming parts reduced from $18.824 \mathrm{~mm}$ to $2.456 \mathrm{~mm}$; in the test, the maximum shape error reduced from $27.26 \mathrm{~mm}$ to $6.03 \mathrm{~mm}$ through the method of die surface modification.

\section{Introduction}

In recent years, lightweight structural design has drawn much attention. Reduction of the weight will not only reduce energy consumption, alleviating the increasingly serious energy crisis, but also reduce environmental pollution caused by exhaust emissions, especially in the automotive and high-speed rail industries [1-3]. Aluminum alloy is widely used in aerospace, rail vehicle manufacturing, and automobile manufacturing as a lightweight material due to its low density, high strength, and ease of recycling [4-6].

With the rapid development of the manufacturing industry, people have more and more diversified requirements on various parts and components, while the parts and components formed by traditional methods have been unable to meet people's needs [7]. As the 3D bending parts can provide a lighter, stiffer, and more space-saving spatial structure than the conventional 2D parts, the demand for $3 \mathrm{D}$ parts is also increasing $[8,9]$. However, the research on the forming method of $3 \mathrm{D}$ parts is still in the exploratory stage.
Hermes et al. developed a machine that specializes in 3D forming of profiles and tubes, in which the combined spinning and bending process leads to low bending forces with the possibility of a significant springback reduction [10]. Gantner et al. used a free-form bending technology to create a freely definable bending geometry, including transitionless bend-in-bends and spline bends [11]. So far, studies about flexible bending are focused on profiles and tubes with rectangular cross section.

In the past decades, stretch bending was the main production method for $2 \mathrm{D}$ parts. At present, it is mainly used in high-volume production of the profile and is also widely used in aircraft, high-speed railway, and automobile and shipbuilding industries [12]. However, the traditional stretch bending is only suitable for mass production rather than small batch production. On one hand, with the improvement of people's living standards, low-cost and small batch production of many kinds of parts and components will be the main way of industrial manufacturing in the future $[13,14]$. On the other hand, it is difficult for complex 
cross-sectional profiles to bend in the horizontal direction and vertical direction without unloading condition through the traditional die $[15,16]$. But the use of the combination of the flexible multipoint forming technology and the stretch bending method can be a good solution to the $3 \mathrm{D}$ bending profile. According to the characteristics of the flexible 3D bending technology, a new flexible 3D stretch bending (FSB) with the multipoint dies is thus being developed. The 3D stretch bending of the profile was successfully achieved.

\section{Flexible 3D Stretch Bending Concept and FE Model}

2.1. Description of the Multipoint Stretch Bending Process. For one thing, the numerical simulation of flexible discrete die forming is more sophisticated than that of the traditional one due to the discontinuous contacts between the profile and the multipoint dies in the multipoint stretch bending (MPSB) process. As shown in Figure 1, the multipoint dies are made up of multiple die units to reconstruct the die surface by changing the space position in the MPSB.

For another, the profile is formed into a 3D part, which requires the profile to be shaped, respectively, in the horizontal direction and vertical direction to bend a spatial contour. Therefore, the MPSB is more diversified than the conventional process due to increased vertical bending of the profile in this process.

A typical MPSB process involves the following stages:

(1) Shape adjustment: In the multipoint dies, the position parameters of each flexible die unit are adjusted to obtain the desired envelope surface.

(2) Prestretching: Under the action of axial tension, the profile is prestretched to a plastic state, and the prestretch can effectively reduce the springback of the forming parts.

(3) Horizontal bending along the $x-y$ plane: The profile is gradually close to the die in the horizontal $x-y$ plane for bending (Figure 2(a)).

(4) Vertical bending along the $x-z$ plane: The multipoint stretch bending machine gradually improves the profile in the vertical $x-z$ plane for bending (Figure 2(b)).

(5) Poststretching: The profile along the tangential direction makes up stretching after the $3 \mathrm{D}$ bending, which can effectively reduce springback of the forming parts.

(6) Releasing: The profile is made of a small amount of plastic after the measurement. Then, the finished product is put into the warehouse.

In the MPSB process, the shape adjustment is based on the spatial contour of the target parts. In the numerical analysis, the stages from (2) to (6) should be simulated to find out the optimal MPSB process of the profile.

2.2. Material Model. Aluminum alloys are commonly used in the field of high-speed railway structures since they offer

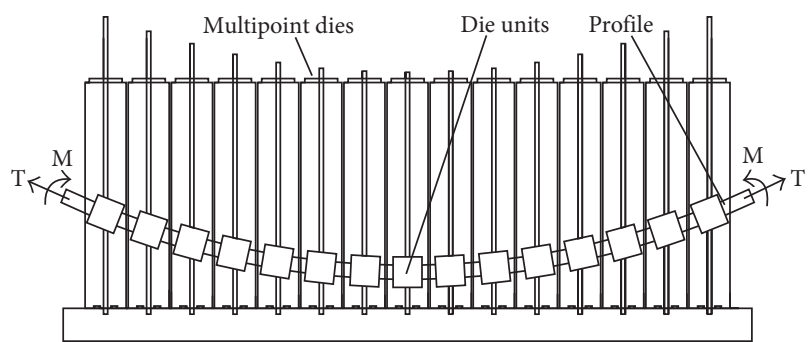

FIGURE 1: Schematic diagram of the multipoint stretch bending machine.

good mechanical properties with low weight [17]. In the numerical investigations, aluminum alloy 6005A is used, which is $6500 \mathrm{~mm}$ in length and about $21 \mathrm{~kg}$ in weight. Meanwhile, isotropic, elastoplastic constitutive behavior with isotropic hardening is assumed for the profile material. The relevant mechanical properties are given as follows: yield strength $\sigma_{\mathrm{y}}=260 \mathrm{MPa}$, elastic modulus $E=70 \mathrm{GPa}$, Poisson's coefficient $v=0.33$, and density $\rho=2.71 \mathrm{~g} / \mathrm{cm}^{3}$. In addition, the preliminary and supplemental tension values are $32.5 \mathrm{~mm}$, and the friction coefficient is 0.1 .

Figure 3 shows the nominal stress $\sigma$ versus nominal strain $\varepsilon$ curve obtained by the authors from the tension test, and this curve was used in the finite element simulation.

2.3. Models of FE Simulation. In the MPSB process, the stages from (2) to (5) should be quasi-static with the explicit, dynamic version of the ABAQUS/Explicit. After stage (5), we used the ABAQUS/Implicit finite element method into numerical simulation to calculate the springback. As shown in Figure 4, the 1/2 finite element model was used to shorten the calculation time and improve the efficiency because the profile is symmetrical along the middle section.

Figure 5 is the parts diagram in the finite element simulation. In this figure, the geometry side of the profile is shown in Figure 6(c). The die units were defined by the plane in contact with the profile, with a length of $50 \mathrm{~mm}$. Their distribution is determined by the horizontal forming condition of the target parts. Meanwhile, the plane plates with the same shape of the die units were defined by the limit screw to bend in the vertical direction for actually controlling the multipoint dies, with a length of $50 \mathrm{~mm}$. Their distribution is determined by the horizontal forming condition and vertical forming condition of the target parts. And the clamps were used with the same shape of the three-dimensional shell structure as the profile, with a length of $100 \mathrm{~mm}$.

The element C3D8R was used to model the aluminum profile. The C3D8R is a hexahedral solid element with eight nodes and linear displacement interpolation, and it is suitable for large strains and deformations.

In the MPSB process, the element R3D4 was used to model the die units, limit screw, and clamp because it did not undergo deformation. The R3D4 is a bilinear quadrilateral three-dimensional rigid element with four nodes. The use of the rigid body element can effectively shorten the calculation time because it does not calculate the unit level in the simulation. 


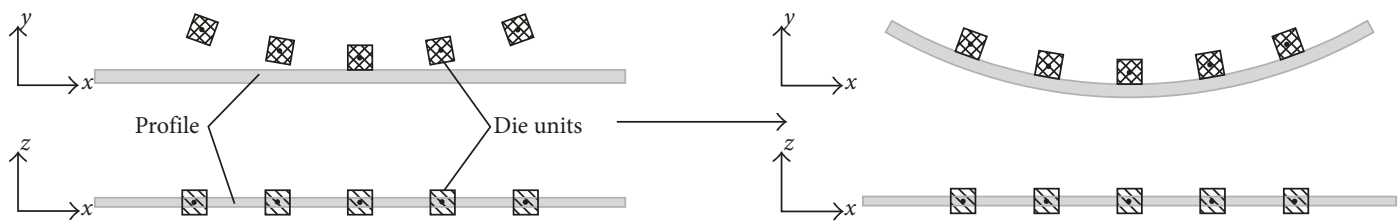

(a)

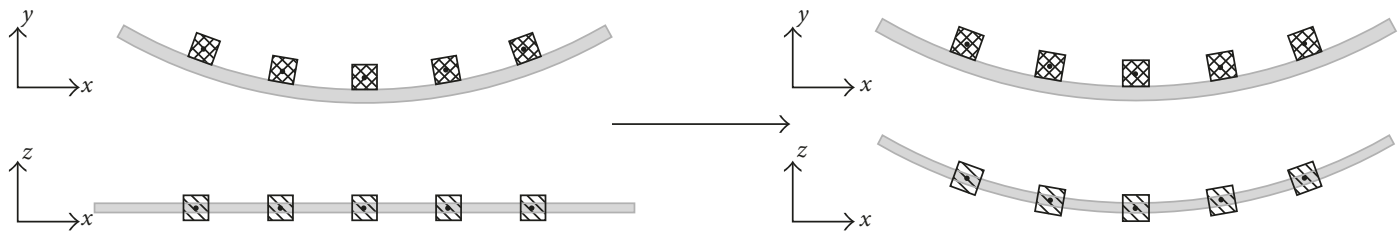

(b)

FIgURE 2: The 3D MPSB process. (a) Horizontal bending along the $x-y$ plane. (b) Vertical bending along the $x-z$ plane.

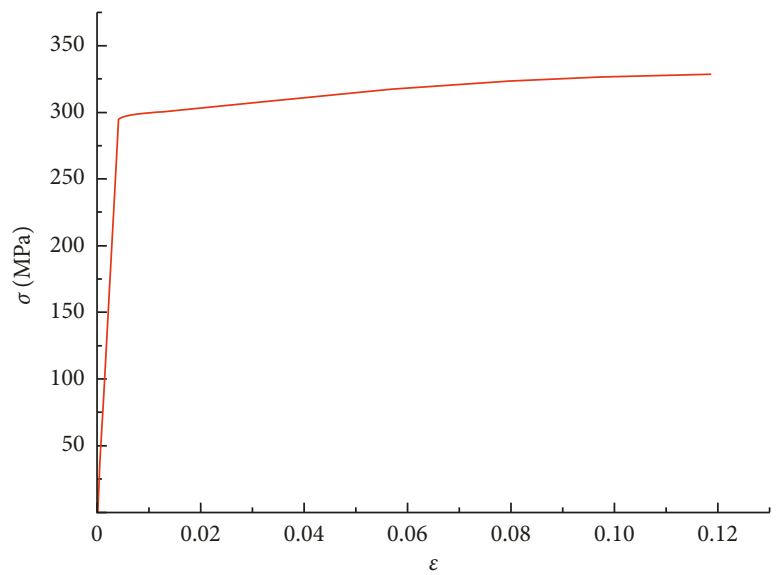

Figure 3: The stress-strain curve of aluminum alloy 6005A.

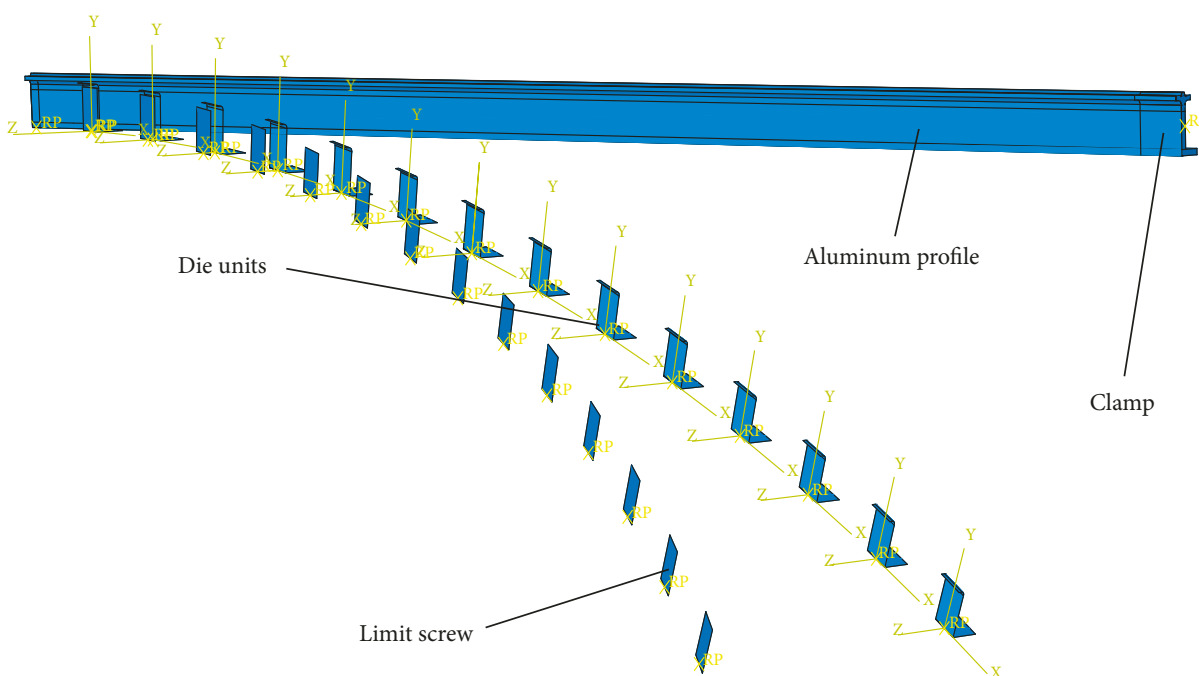

FIGURE 4: Finite element models for flexible 3D stretch bending.

In the finite element simulation, the finite element model is divided into four submodels and six analytic steps, which are defined as the horizontal bending model, vertical bending model, poststretching model, and springback model, respectively. The horizontal bending model includes the initial analysis step, the prestretching process, and the 


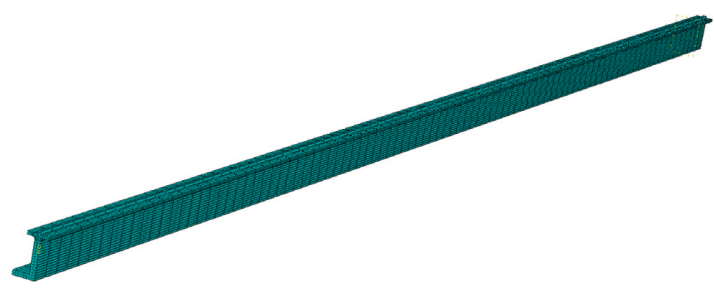

(a)

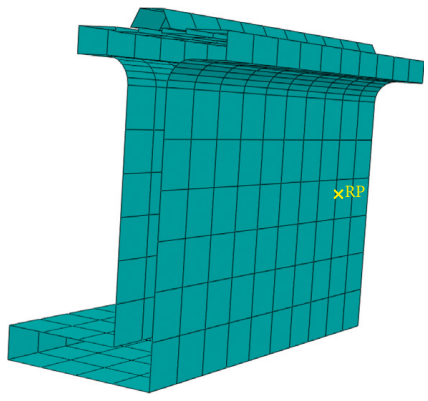

(c)

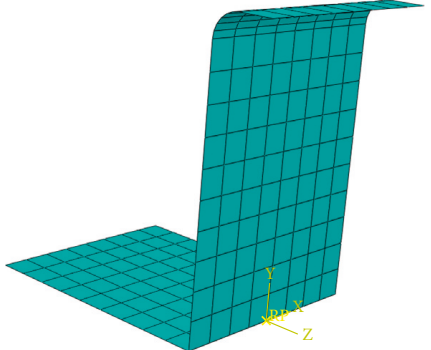

(b)

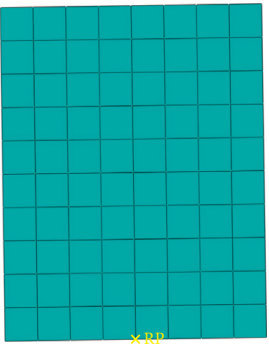

(d)

Figure 5: The parts diagram. (a) Aluminum profile. (b) Die units. (c) Clamp. (d) Limit screw.

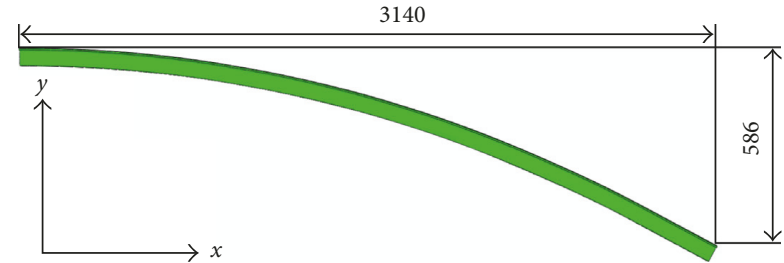

(a)

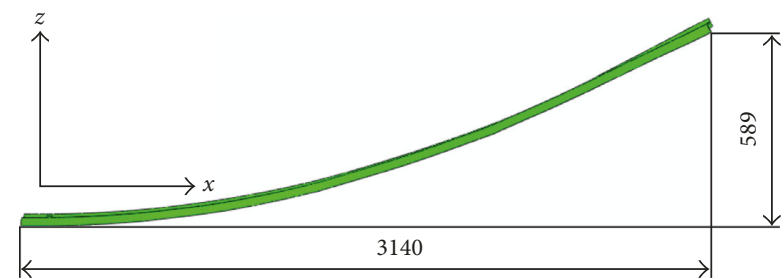

(b)

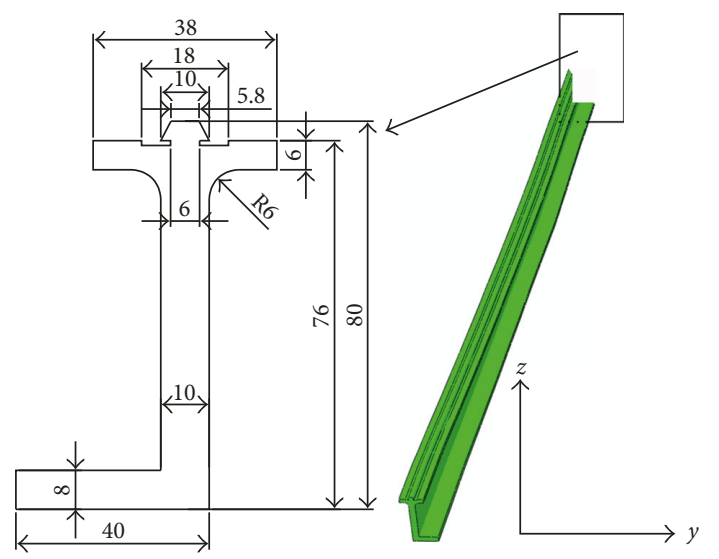

(c)

FIGURE 6: The 3D stretch bending parts. (a) Vertical view. (b) Front view. (c) Right view and the geometry size of the profile. horizontal bending stage; the vertical bending model includes the vertical bending stage; the poststretching model includes the poststretching process; and the springback model includes the springback process. After each model task completes successfully, it is used as a predefined field to start the next model run.

\section{Numerical Analysis of the Shape Accuracy in the MPSB}

In the MPSB process, the number of die units not only affects the manufacturing cost of $3 \mathrm{D}$ forming parts but also causes its shape accuracy, stress, and strain to change. Therefore, in the MPSB process, it is vital to choose the number of die units. Figure 6 shows the parameters of the aluminum profile in the $3 \mathrm{D}$ stretch bending. According to these parameters, the four options of the number of die units $m=15, m=20$, $m=25$, and $m=30$ are chosen to simulate the whole MPSB process in order to determine the most suitable die units of the $3 \mathrm{D}$ process in the numerical investigation.

3.1. Effect of the 3D Stretch Bending on Shape Accuracy. Figure 7 shows the maximum shape error between the forming parts and the target parts with different numbers of die units after the vertical bending. With the increasing number of die units, the maximum shape error of the forming parts will be reduced. This is mainly due to the fact that the displacement amplitude and clearance value of adjacent dies become smaller with more die units. Therefore, the shape accuracy of the forming parts can be effectively improved.

Figure 8 is the graph of the equivalent stress distribution after the 3D stretch bending of different numbers of die units. As shown in Figure 8(a), the apparent wrinkle of the aluminum profile affects the shape accuracy of the forming 


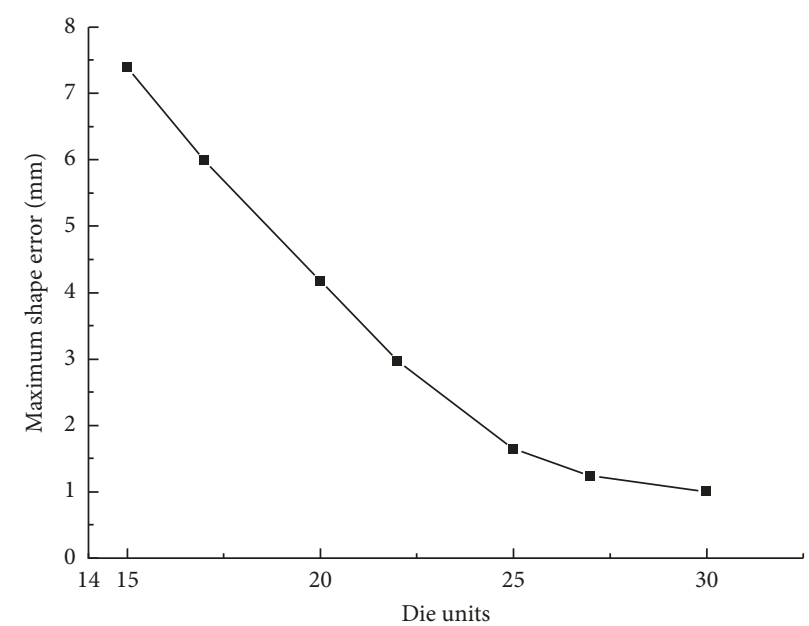

Figure 7: The maximum shape error between the forming parts and the target parts.
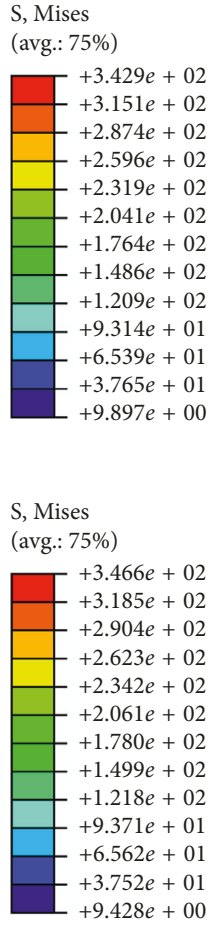

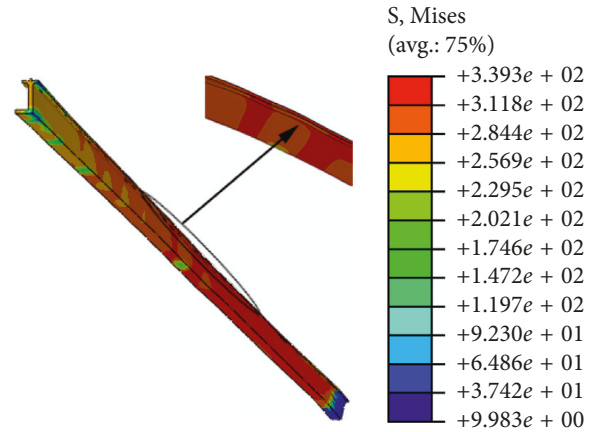

(a)

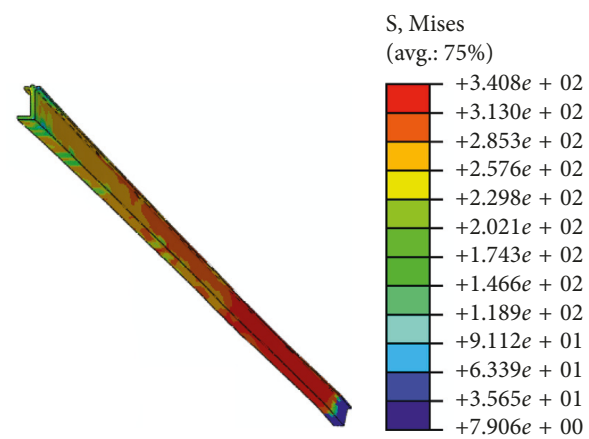

(c)

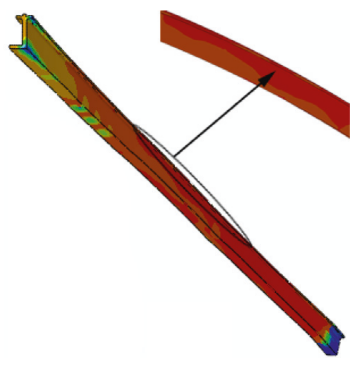

(b)

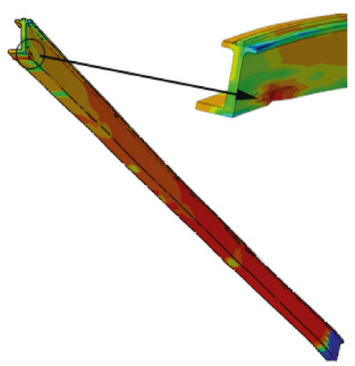

(d)

FIgURE 8: The equivalent stress distribution of the profile after the vertical bending along the $x$ - $z$ plane: (a) the number of die units: $m=15$, (b) the number of die units: $m=20$, (c) the number of die units: $m=25$, and (d) the number of die units: $m=30$.

parts after the vertical bending, when the number of die units is 15 . As the number of die units increases, its wrinkle is significantly reduced (as shown in Figure 8(b)).

However, with the increasing number of die units, the manufacturing cost of the forming parts will also increase. When the number of die units increases to a certain amount, the profile will undergo depression (as shown in Figure 8(d)). Therefore, selecting the appropriate number of die units is the precondition for the bending quality of the forming parts.

At the end of the aluminum profile, that is, at the clamp on the force-applying end of the profile, the stress-strain field undergoes a drastic change due to the concentrated load on the clamp. Thus, it is generally used as the stub bar to handle, or it adopts flexible clamping machinery to control the practical production process.

3.2. Effect of the Springback on Shape Accuracy. In the bending process, the defects often affect the quality of the forming parts, such as springback, wrinkles, and fracture. The inevitable factor is the springback, which is closely related to the entire bending process. The reduction of the 


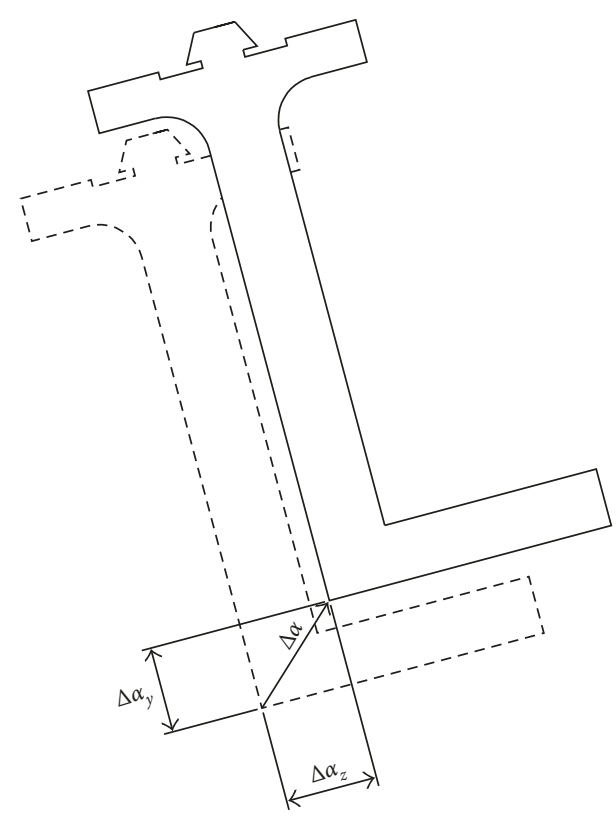

FIgURE 9: The front and back spatial positions of the end face contours.

springback can effectively improve the shape accuracy of the forming parts. Therefore, the research on springback is indispensable in the MPSB process. For the study of the profile springback, we adopt the predefined field in ABAQUS/Standard and delete the redundant constraint. It is only observed that the springback phenomenon is caused by the stress-strain field after the poststretching.

The forming process is more complicated than the traditional bending process because the aluminum profile is formed along the spatial curve in the MPSB process. Therefore, the springback process is divided into horizontal direction along the $x-y$ plane and vertical direction along the $x-z$ plane, as shown in Figure 9 , where $\Delta \alpha$ represents the total springback value, $\Delta \alpha_{y}$ represents the springback value along the $x-y$ plane, and $\Delta \alpha_{z}$ represents the springback value along the $x-z$ plane.

Figure 10 shows $\Delta \alpha$ with the change of twist angle after the MPSB process. As shown in the figure, with the increase of twist angle for the profile, the springback of the forming parts also increases in the profile section under the action of the die units. Thus, controlling the profile deformation within a certain range can effectively reduce the springback in the MPSB process. At the same time, the curves in Figure 10 clearly demonstrate that the final springback value of the aluminum profile reduces when the number of die units increases.

Figures 11(a) and 11(b) show the horizontal and vertical springback values of the profile along the length. It can be observed from these results that the horizontal springback value and the vertical springback value also decrease when the number of die units increases. At the same time, we can find that the springback value in the vertical direction is smaller than that in the horizontal direction. This is because the aluminum profile has been bent horizontally along the $x-y$ plane before the vertical bending. After the horizontal bending, the stress and strain fields of the aluminum profile

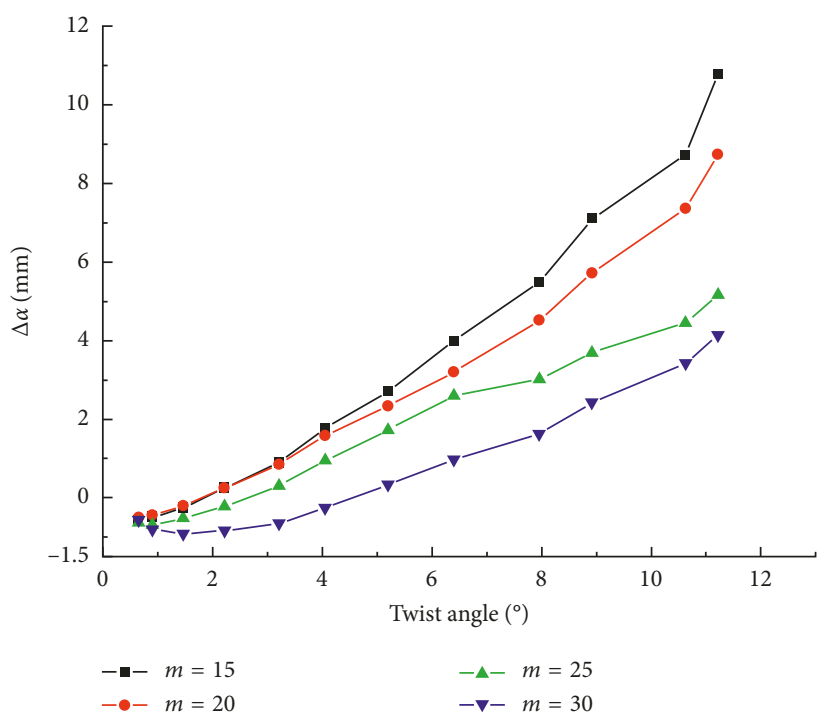

FIGURE 10: The total springback value of the profile with the change of twist angle.

change. The superposition of the stress and strain fields causes the springback value to be larger in the horizontal direction than that in the vertical direction.

In summary, it can effectively reduce the springback value of the profile with the increase of the number of die units. However, with the increase of die units, it will not only increase the manufacturing cost of the forming parts but also make the profile undergo depression (as shown in Figure $8(\mathrm{~d})$ ). Therefore, it is very important to choose the suitable number of die units in the MPSB process.

3.3. The Method Based on Reverse Compensation of the Springback Value. After the MPSB process, the shape error of the profile is mainly due to the springback along the $x-y$ horizontal plane and the $x-z$ vertical plane. Therefore, we used the following method based on reverse compensation of the springback value to modify the die units' surface. Firstly, according to the discrete feature of the multipoint dies, the springback value of the profile was simulated by ABAQUS. Then, the springback value was compensated to each die unit to modify the dies' surface. Finally, the shape error was controlled in the range of the accuracy requirement after multiple iterations.

Figure 12 shows the contrast of the curved surface shape before and after the adjustment in the $x$ - $y$ horizontal plane, where $\Delta \theta_{i}^{j}$ represents the measured springback error, $\Delta \eta_{i}^{j}$ represents the displacement of the springback compensation, the superscript $j$ represents the number of iterations, and the subscript $i$ represents the number of shape control points. In addition, the adjustment method along the $x-z$ vertical plane is similar to that along the $x-y$ horizontal plane. Moreover, the initial adjusting method of the die units takes the shape of the target part as the multipoint dies' envelope surface.

After the springback compensation, the die units' envelope surface is calculated as follows: 


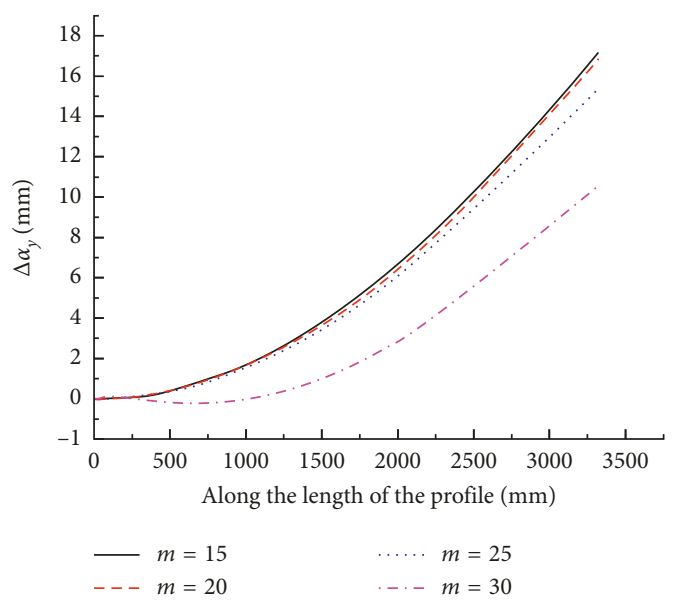

(a)

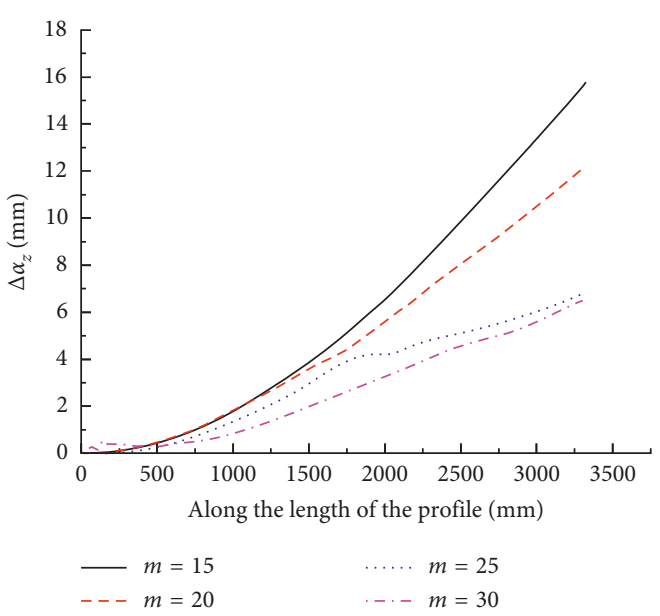

(b)

FIGURE 11: The springback value along the longitudinal direction of the profile. (a) The springback value along the $x$ - $y$ plane. (b) The springback value along the $x-z$ plane.

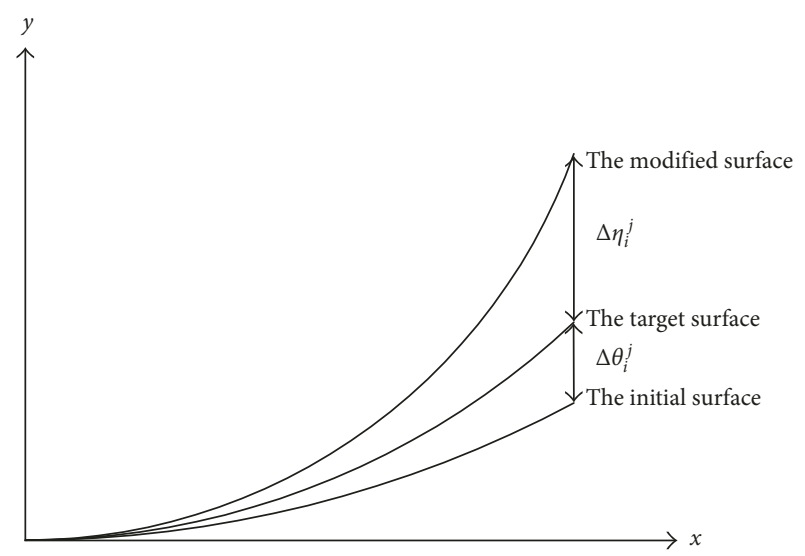

FIgURE 12: Schematic diagram before and after the adjustment along the $x-y$ horizontal plane.

$$
\begin{aligned}
K_{i}^{j+1} & =K_{i}^{j}+\Delta \eta_{i}^{j}, \\
\Delta \eta_{i}^{j} & =\alpha \Delta \theta_{i}^{j},
\end{aligned}
$$

where $\alpha$ represents the compensation factor of the springback, the value of which is generally $[-2.5,-1]$, and $K$ represents the die units' envelope shape.

First, through the ABAQUS finite element simulation, we got the springback values along the $x-y$ horizontal plane and the $x-z$ vertical plane when the number of die units was 25 and then reversed the compensation to each die unit to get the discrete point information. Next, according to this information, the theoretical curves of the horizontal and vertical lines of the forming part were obtained by the fourth-degree polynomial fit.

The theoretical curve of the horizontal line along the $x-y$ plane is as follows:

$$
\begin{aligned}
Y= & -0.29477-0.00466 X+9.50531 \times 10^{-5} X^{2} \\
& -4.2123 \times 10^{-9} X^{3}+1.48643 \times 10^{-12} X^{4} .
\end{aligned}
$$

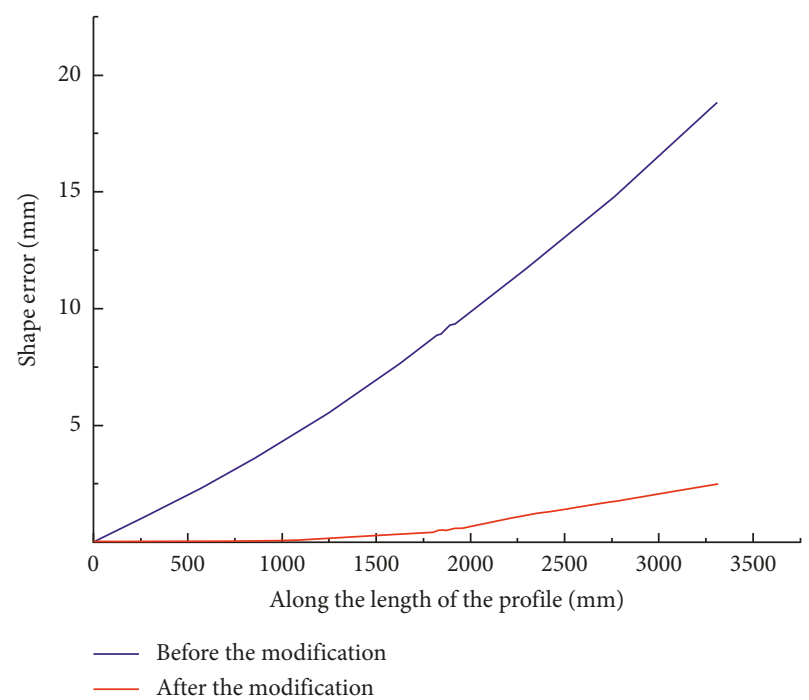

FIGURE 13: The shape error before and after the modification.

The theoretical curve of the vertical line along the $x-z$ plane is as follows:

$$
\begin{aligned}
Z= & -0.17864-0.00614 X+9.79094 \times 10^{-5} X^{2} \\
& -6.15386 \times 10^{-9} X^{3}+1.80589 \times 10^{-12} X^{4} .
\end{aligned}
$$

Moreover, the die units' envelope surface was again adjusted by the theoretical curve. Finally, the ABAQUS finite element software was used to simulate the MPSB process according to the forming parameters after the shape adjustment to obtain the shape error value of the forming parts. The curves in Figure 13 clearly demonstrate that it can effectively reduce the shape error between the forming parts and the target parts after the springback by modifying the die units' envelope surface. The die units' surface modification method can reduce the shape error of the forming parts 


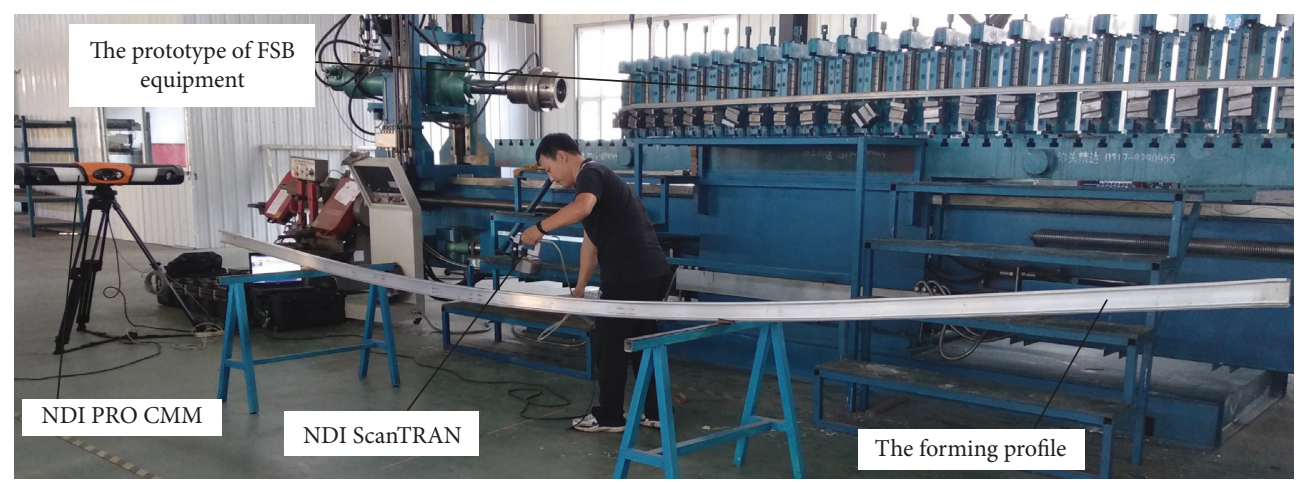

FIgURE 14: The detection setup and the forming part through the MPSB process.

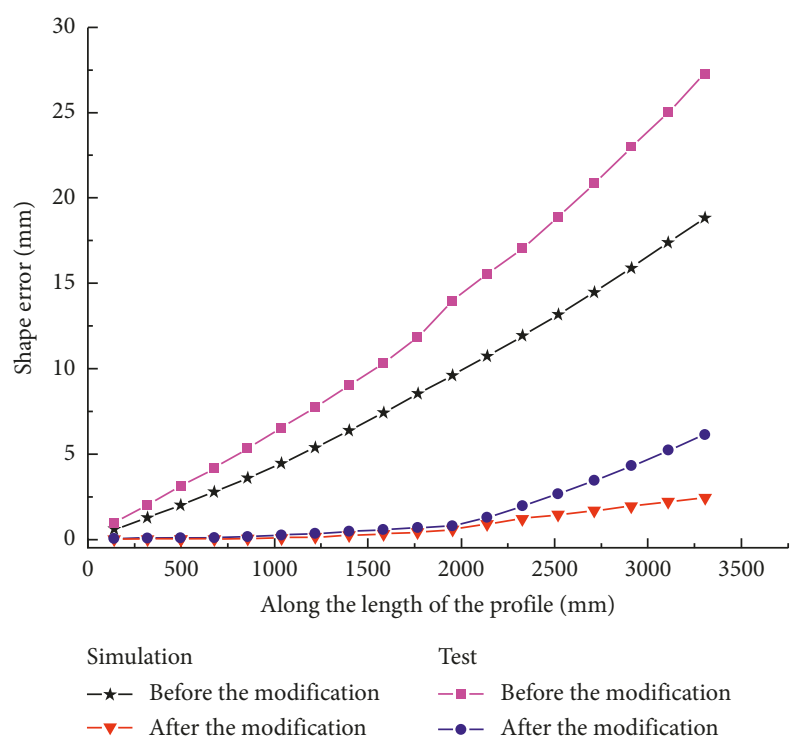

FIGURE 15: Comparison of the test results and the numerical simulation results.

caused by the springback and thus improve the shape accuracy of the forming process.

\section{The Shape Accuracy Test of the Forming Part}

In this paper, the flexible 3D multipoint stretch bending test was carried out to verify the reliability of the simulation, taking the frame of a high-speed railway car as an example (as shown in Figure 14). The test process was shown as follows: First of all, we used the prototype of flexible 3D stretch bending (FSB) equipment to make $3 \mathrm{D}$ bending of the aluminum profile and then scanned the forming profile by using the NDI large space measuring instrument PRO CMM 3500 optical tracker. Finally, the scanned 3D map of the forming part and the target part was 3D analyzed with the Geomagic Qualify software. Figure 15 is the comparison between the test results and the numerical simulation results. It can be seen that the test results are in agreement with the numerical simulation results. Moreover, the shape error between the forming part and the target part can be significantly reduced, and the shape accuracy of the forming part can be improved by using the method of die surface modification.

\section{Conclusion}

(1) In the MPSB, less die units will reduce the forming accuracy of the profile, while more die units can reduce the springback of the profile. But more die units will increase the manufacturing cost of the aluminum profile at the same time. Therefore, it is very important to select the appropriate number of die units in the MPSB process. In this paper, 25 was determined as the ideal number of die units.

(2) The $x-y$ horizontal and $x-z$ vertical springback compensations are performed for the complex section profile. In the numerical simulation, the maximum shape error reduced from $18.824 \mathrm{~mm}$ to $2.456 \mathrm{~mm}$; in the test, the maximum shape error reduced from $27.26 \mathrm{~mm}$ to $6.03 \mathrm{~mm}$ by the method of die surface modification. Therefore, the method of die surface modification is an effective way to improve the shape accuracy of the forming parts.

(3) The 3D MPSB test for the profile was carried out. The test results showed that they are in agreement with the simulation results. For this purpose, the forming parameters can be optimized by the finite element simulation software to guide the production test and reduce the production cost.

\section{Conflicts of Interest}

The authors declare that they have no conflicts of interest.

\section{Acknowledgments}

This work was supported by the National Natural Science Foundation of China (51675225).

\section{References}

[1] H. Z. Li, G. Y. Sun, G. Li, Z. Gong, D. Liu, and Q. Li, “On twist springback in advanced high-strength steels," Materials \& Design, vol. 32, no. 6, pp. 3272-3279, 2011. 
[2] C. G. Liu, X. G. Zhang, X. T. Wu, and Y. Zheng, "Optimization of post-stretching elongation in stretch bending of aluminum hollow profile," International Journal of Advanced Manufacturing Technology, vol. 82, no. 9-12, pp. 1737-1746, 2015.

[3] R. Y. Zhang, G. Y. Zhao, Z. H. Gou, and Y. P. Quan, "Effects of material parameters on springback of 5052 aluminium alloy sections with hat profile in rotary draw bending," International Journal of Advanced Manufacturing Technology, vol. 80, no. 5-8, pp. 1067-1075, 2015.

[4] S. P. Shanmuganatan and V. S. Senthil Kumar, "Experimental investigation and finite element modeling on profile forming of conical component using $\mathrm{Al}$ 3003(O) alloy," Materials \& Design, vol. 36, pp. 564-569, 2012.

[5] T. J. Liu, Y. J. Wang, J. J. Wu et al., "Springback of extruded 2196-T8511 and 2099-T83 Al-Li alloys in stretch bending," Procedia Engineering, vol. 81, pp. 981-986, 2014.

[6] T. J. Liu, Y. J. Wang, J. J. Wu et al., "Springback analysis of Z \& T-section 2196-T8511 and 2099-T83 Al-Li alloys extrusions in displacement controlled cold stretch bending," Journal of Materials Processing Technology, vol. 225, pp. 295-309, 2015.

[7] J. C. Liang, S. Gao, F. Teng, P. Z. Yu, and X. J. Song, "Flexible 3D stretch-bending technology for aluminum profile," International Journal of Advanced Manufacturing Technology, vol. 71, no. 9-12, pp. 1939-1947, 2014.

[8] F. Teng, W. X. Zhang, J. C. Laing, and S. Gao, "Springback prediction and optimization of variable stretch force trajectory in three-dimensional stretch bending process," Chinese Journal of Mechanical Engineering, vol. 28, no. 6, pp. 11321140, 2015.

[9] M. Wang, Z. N. Liu, G. L. Lu, and Z. Y. Cai, "Analysis of continuous roll forming for manufacturing 3D surface part with lateral bending deformation," International Journal of Advanced Manufacturing Technology, vol. 93, no. 5-8, pp. 2251-2261, 2017.

[10] M. Hermes, D. Staupendahl, C. Becher, and A. E. Tekkaya, "Innovative machine concepts for 3D bending of tubes and profiles," Key Engineering Materials, vol. 473, pp. 37-42, 2011.

[11] P. Gantner, H. Bauer, D. K. Harrison, and A. K. M. De Silva, "Free-bending-a new bending technique in the hydroforming process chain," Journal of Materials Processing Technology, vol. 167, no. 2-3, pp. 302-308, 2005.

[12] S. H. Wang, Z. Y. Cai, M. Z. Li, and Y. W. Lan, "Numerical simulation on the local stress and local deformation in multipoint stretch forming process," International Journal of Advanced Manufacturing Technology, vol. 60, no. 9-12, pp. 901-911, 2011.

[13] M. Takamura, M. Sakata, A. Fukui et al., "Investigation of twist in curved hat channel products by elastic-plastic finite element analysis," International Journal of Material Forming, vol. 3, no. S1, pp. 131-134, 2010.

[14] S. L. Zang, M.-G. Lee, L. Sun, and J. H. Kim, "Measurement of the Bauschinger behavior of sheet metals by three-point bending springback test with pre-strained strips," International Journal of Plasticity, vol. 59, pp. 84-107, 2014.

[15] S. Chatti, M. Hermes, A. E. Tekkaya, and M. Kleiner, "The new TSS bending process: $3 \mathrm{D}$ bending of profiles with arbitrary cross-sections," CIRP Annals-Manufacturing Technology, vol. 59, no. 1, pp. 315-318, 2010.

[16] P. H. Vatter and R. Plettke, "Process model for the design of bent 3-dimensional free-form geometries for the three-rollpush-bending process," Procedia CIRP, vol. 7, pp. 240-245, 2013.
[17] Z. Y. Cai, S. H. Wang, X. D. Xu, and M. Z. Li, "Numerical simulation for the multi-point stretch forming process of sheet metal," Journal of Materials Processing Technology, vol. 209, no. 1, pp. 396-407, 2009. 


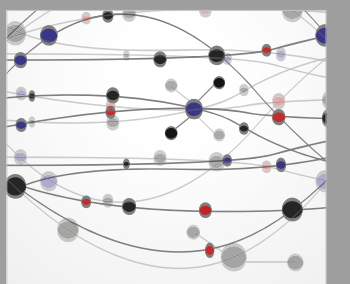

The Scientific World Journal
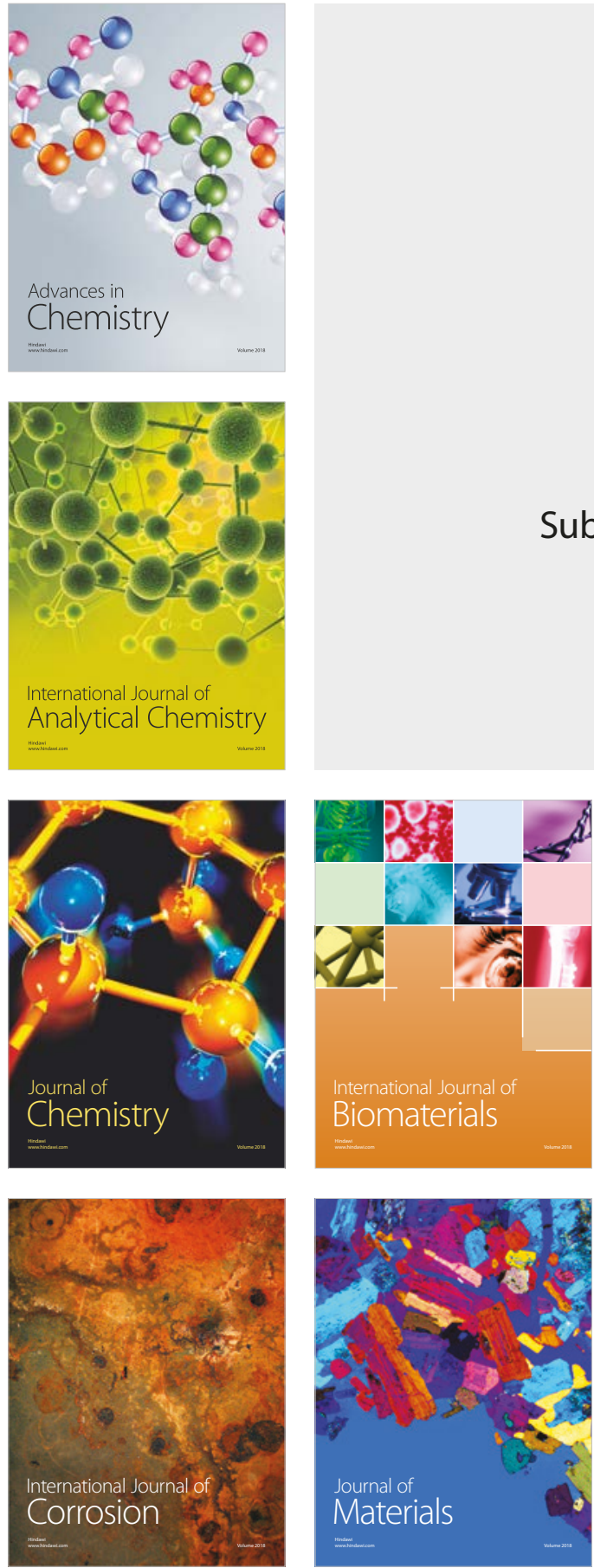

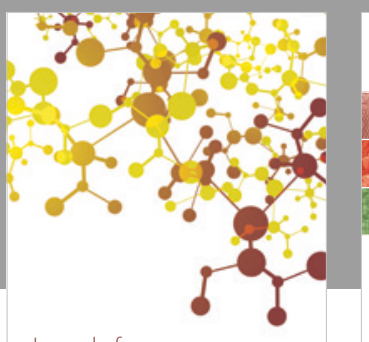

Journal of

Applied Chemistry
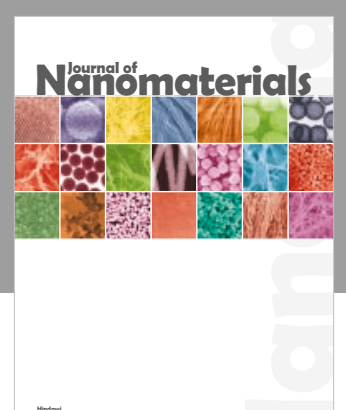

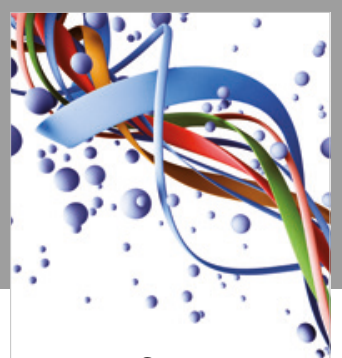

Scientifica

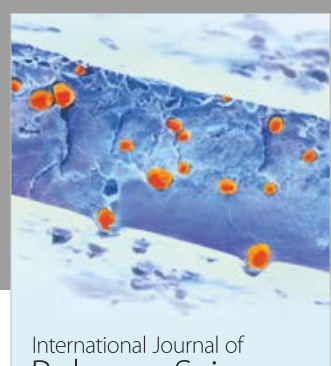

Polymer Science

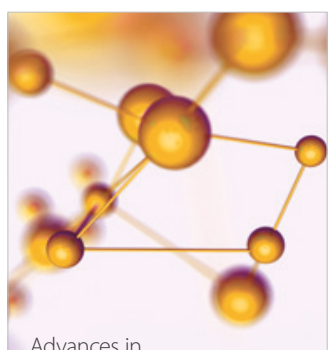

Physical Chemistry
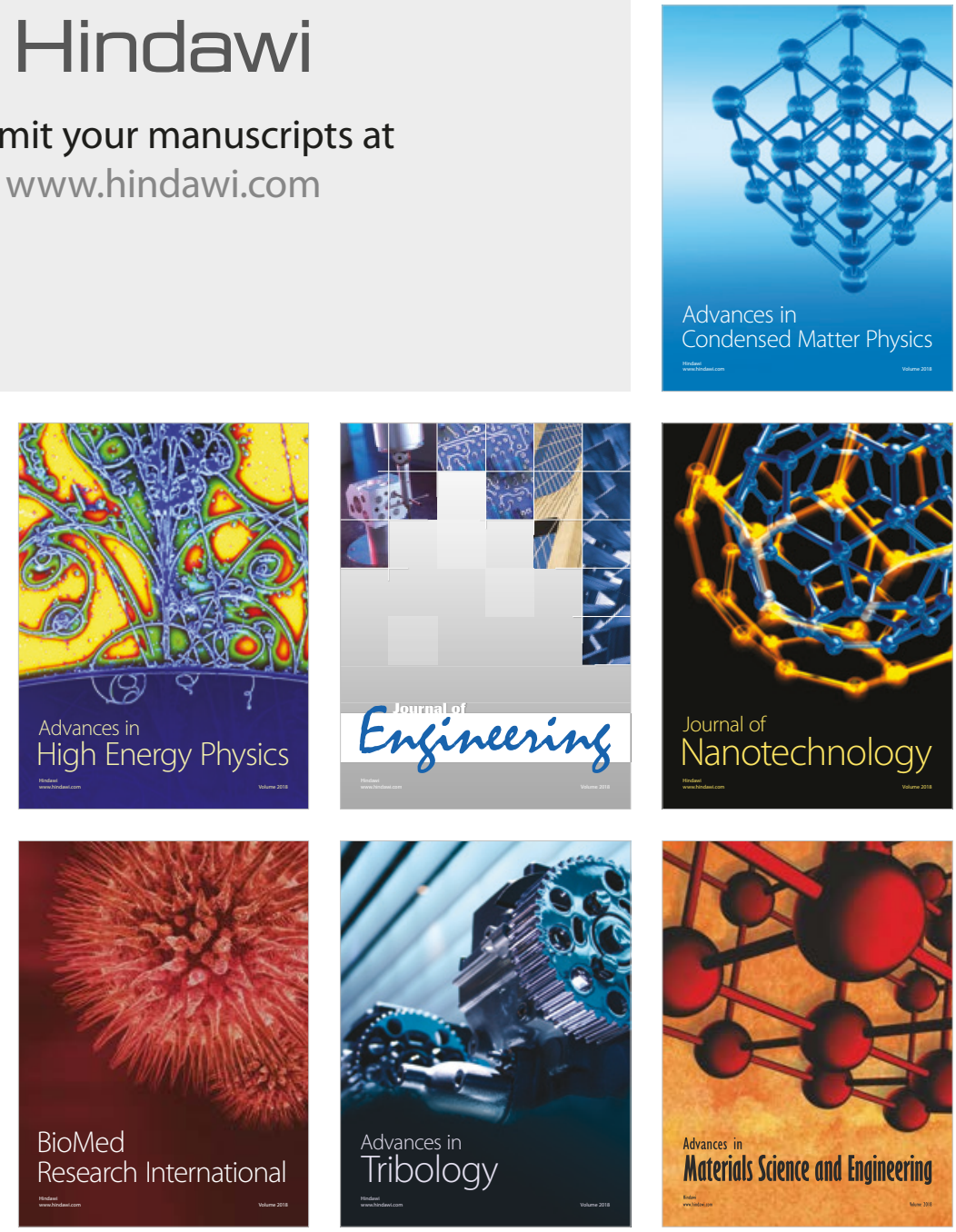